\title{
ATUAÇÃO FISIOTERAPÊUTICA EM ADULTOS SUBMETIDOS AO SUPORTE DE VIDA COM OXIGENAÇÃO POR MEMBRANA EXTRACORPÓREA
}

\author{
PHYSIOTHERAPY PERFORMANCE IN ADULTS SUBMITTED TO LIFE SUPPORT WITH \\ OXYGENATION BY EXTRACORPOREAL MEMBRANE
}

DOI: $10.16891 / 2317-434 X . v 10 . e 1 . a 2022 . p p 1251-1254$

Recebido em: 10.11 .2021 | Aceito em: 10.11.2021
Poliana da Silva Almeida, Antônia Mayara Oliveira de Freitas, Francisco Emerson Alves da Silva e Anny Karolliny Pinheiro de Sousa Luz

Centro Universitário Doutor Leão Sampaio

\begin{abstract}
RESUMO
A oxigenação por membrana extracorpórea (ECMO) é um suporte de assistência circulatória e/ou respiratória, indicada para pacientes que desenvolvem falência respiratória hipoxêmica refratária e/ou complicações cardiovasculares graves. Os pacientes submetidos ao ECMO são afetados pelo tempo prolongado de imobilização e internação, reduzindo a capacidade funcional e subsequentes prejuízos à qualidade de vida. O presente estudo tem como propósito ampliar os conhecimentos sobre a atuação do fisioterapeuta no manejo dos pacientes submetidos ao suporte de vida por ECMO. Este trabalho trata-se de uma revisão integrativa realizado nas principais bases de dados: PEDro, Lilacs, Scielo e PubMed, com os DeCs: Physiotherapy/ Extracoporeal membrane oxygenation/ ECMO/ Mobilização precoce/ Fisioterapia nas línguas portuguesas e inglesas com o operador booleano AND. Para critério de inclusão, foram escolhidos os periódicos publicados nos últimos 5 anos (2017-2021) em idioma inglês, português e espanhol. Os estudos indicam que as condutas fisioterapêuticas realizadas em adultos em suporte de vida por ECMO, são seguras e podem reduzir o tempo de internação na unidade de terapia intensiva (UTI), além de apresentar maior chance de retorno às atividades de vida diária. Mediante o exposto, a fisioterapia motora e respiratória deve ser empregue em pacientes durante a ECMO. Pacientes submetidos a reabilitação precoce sofrem menos risco de desenvolver delirium, reduzem o tempo em ventilação mecânica invasiva (VMI), diminuem os efeitos deletérios da imobilização prolongada e têm menos prejuízos à capacidade funcional.
\end{abstract}

Palavras-chave: Suporte de vida; Mobilização precoce; Fisioterapia motora.

\section{ABSTRACT}

Extracorporeal membrane oxygenation (ECMO) is a support for circulatory and/or respiratory assistance, indicated for patients who develop refractory hypoxemic respiratory failure and/or severe cardiovascular complications. Patients undergoing ECMO are affected by the prolonged time of immobilization and hospitalization, reducing functional capacity and subsequent damage to quality of life. The present study aims to increase knowledge about the role of physical therapists in the management of patients undergoing ECMO life support. This work is an integrative review carried out in the main databases: PEDro, Lilacs, Scielo and PubMed, with the DeCs: Physiotherapy/ Extracoporal membrane oxygenation/ ECMO/ Early mobilization/ Physiotherapy in Portuguese and English with the Boolean operator AND. For inclusion criteria, journals published in the last 5 years (2017-2021) in English, Portuguese and Spanish were chosen. Studies indicate that physiotherapeutic conducts performed in adults on ECMO life support are safe and can reduce the length of stay in the intensive care unit (ICU), in addition to presenting a greater chance of returning to activities of daily living. Given the above, motor and respiratory physiotherapy should be used in patients during ECMO. Patients undergoing early rehabilitation are at less risk of developing delirium, reduce the time on invasive mechanical ventilation (IMV), reduce the deleterious effects of prolonged immobilization and have less damage to functional capacity.

Keyword: Life support; Early mobilization; Motor physiotherapy. 


\section{V.10 N.1 (2022) ISSN: 2317-434X}

\section{INTRODUÇÃOO}

A oxigenação por membrana extracorpórea (ECMO) é uma modalidade de suporte de vida indicada em casos de falência pulmonar e/ou cardíaca refratária às medidas convencionais. Este tipo de assistência à vida é indicado para pacientes com alto risco que apresentam Síndrome do Desconforto Respiratório Agudo (SDRA), choque cardiogênico refratário ou, até mesmo, parada cardiorrespiratória (LAGES; TIMENETSKY, 2021).

A ECMO integra um sistema heparinizado que é instalado percutaneamente em uma veia, onde o sangue é drenado e bombeado por meio de uma bomba centrífuga para uma membrana oxigenadora. Ocorre o processo de troca gasosa, troca de oxigênio pelo gás carbônico na membrana oxigenadora, e o sangue oxigenado é devolvido ao paciente através de um sistema venoso ou arterial (NAKASATO; LOPES; LOPES, 2018).

Os pacientes submetidos ao ECMO sofrem os efeitos deletérios da imobilização prolongada, a longa internação acarreta diminuição da massa muscular em alto grau, fraqueza muscular generalizada, redução da capacidade funcional, danos psicológicos, afetando significativamente a qualidade de vida. A fisioterapia visa diminuir os efeitos da longa permanência na UTI com protocolos bem definidos de mobilização precoce para reabilitar, prevenir ou reduzir a perda de massa muscular e força, entre outros benefícios (FERREIRA et al., 2018).

Diante do exposto, esse estudo tem como objetivo ampliar os conhecimentos sobre atuação do fisioterapeuta no manejo dos pacientes submetidos ao suporte de vida por ECMO, visando entender os benefícios da fisioterapia e os cuidados que a equipe multidisciplinar deve ter com esses pacientes críticos.

\section{METODOLOGIA}

Trata-se de uma revisão integrativa acerca do papel da fisioterapia em pacientes com suporte de vida por ECMO. Foram incluídos no estudo artigos disponíveis gratuitamente, publicados no período de 2017 a 2021, em idiomas inglês, português e espanhol, que possuíam no título os descritores referentes ao trabalho. Foram excluídos estudos de caso, relatos de experiência, revisões narrativas e artigos não compatíveis com a questão norteadora.

Foi realizado uma busca nas principais bases de dados: Physiotherapy Evidence Database (PEDro), Scientific Eletronic Library Online (Scielo), Public Medline (PubMed), Literatura Latino Americana e do Caribe em Ciências da Saúde (LILACS), utilizando os Descritores em Ciência da Saúde (DeCS): Physiotherapy/

\section{Extracoporeal membrane oxygenation/ ECMO/} Mobilização precoce/ Fisioterapia/ Suporte de Vida/ Paciente crítico associado ao operador boleano AND para cruzar esses descritores.

Os descritores foram associados da seguinte forma: Extracoporeal membrane oxygenation and Physiotherapy; Mobilização precoce and paciente crítico; Suporte de vida and ECMO; Fisioterapia and mobilização precoce; Fisioterapia and oxigenação por membrana extracorpórea.

\section{RESULTADOS E DISCUSSÕES}

Foram encontrados 17 (dezessete) artigos nas bases de dados, porém apenas 8 (oito) periódicos preencheram os critérios de inclusão após uma revisão detalhada. A descrição da busca dos artigos foi descrita no fluxograma 1.

Dos 8 (oito) artigos escolhidos, 6 (seis) autores não relataram complicações relacionados à realização da fisioterapia associada ao ECMO. Carswell et al. (2017) relataram queda da saturação periférica de oxigênio durante a mobilização, mas essa queda foi compensada pelo aumento do fluxo sanguíneo da ECMO. Segundo Nakataso et al. (2020) o posicionamento inadequado do paciente pode obstruir o fluxo contínuo da ECMO ou até mesmo deslocar a cânula (femoral ou jugular), essas situações podem causar hipoxemia grave. Apenas um estudo demonstrou evento adverso durante as intervenções, porém sem complicações severas.

Todos os trabalhos evidenciaram redução significativa na mortalidade dos pacientes que foram submetidos às condutas fisioterapêuticas. Dois estudos demonstraram que o tempo de ventilação mecânica invasiva (VMI) reduziu para os pacientes que tiveram intervenção da fisioterapia quando comparado com pacientes que não tiveram.

De acordo com Nakataso et al. (2017) a mobilização e a reabilitação dos pacientes em ECMO reduziu drasticamente o tempo de internação hospitalar e internação em unidade de terapia intensiva desses pacientes. Rehder et al. (2019) relatou em seu estudo que o tempo médio de internação hospitalar dos pacientes do grupo controle foi de 45 dias e no grupo intervenção foi de 11 dias.

Os estudos reportaram que a fisioterapia, além dos benefícios gerais citados anteriormente, também melhora a capacidade respiratória e funcional, melhora o trofismo e força muscular, favorece a eliminação de secreções e diminui as complicações associadas ao imobilismo.

De acordo com os autores, a fisioterapia não é contraindicada em pacientes que estão em ECMO, pelo contrário, as condutas fisioterápicas devem ser 


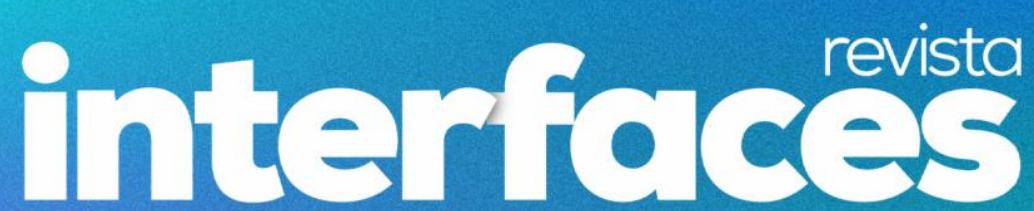

\section{V.10 N.1 (2022) ISSN: $2317-434 X$}

implementadas precocemente, desde que a equipe multidisciplinar seja especializada e respeite as condições clínicas do paciente para maior segurança. É necessário planejamento, cuidado e acompanhamento durante todo o processo, os profissionais devem ser capacitados e experientes na área.

Figura 1. Fluxograma do processo de seleção para inclusão dos artigos. uma busca nas principais bases de dados: Physiotherapy Evidence Database (PEDro), Scientific Eletronic Library Online (Scielo), Public Medline (PubMed), Literatura Latino Americana e do Caribe em Ciências da Saúde (LILACS).

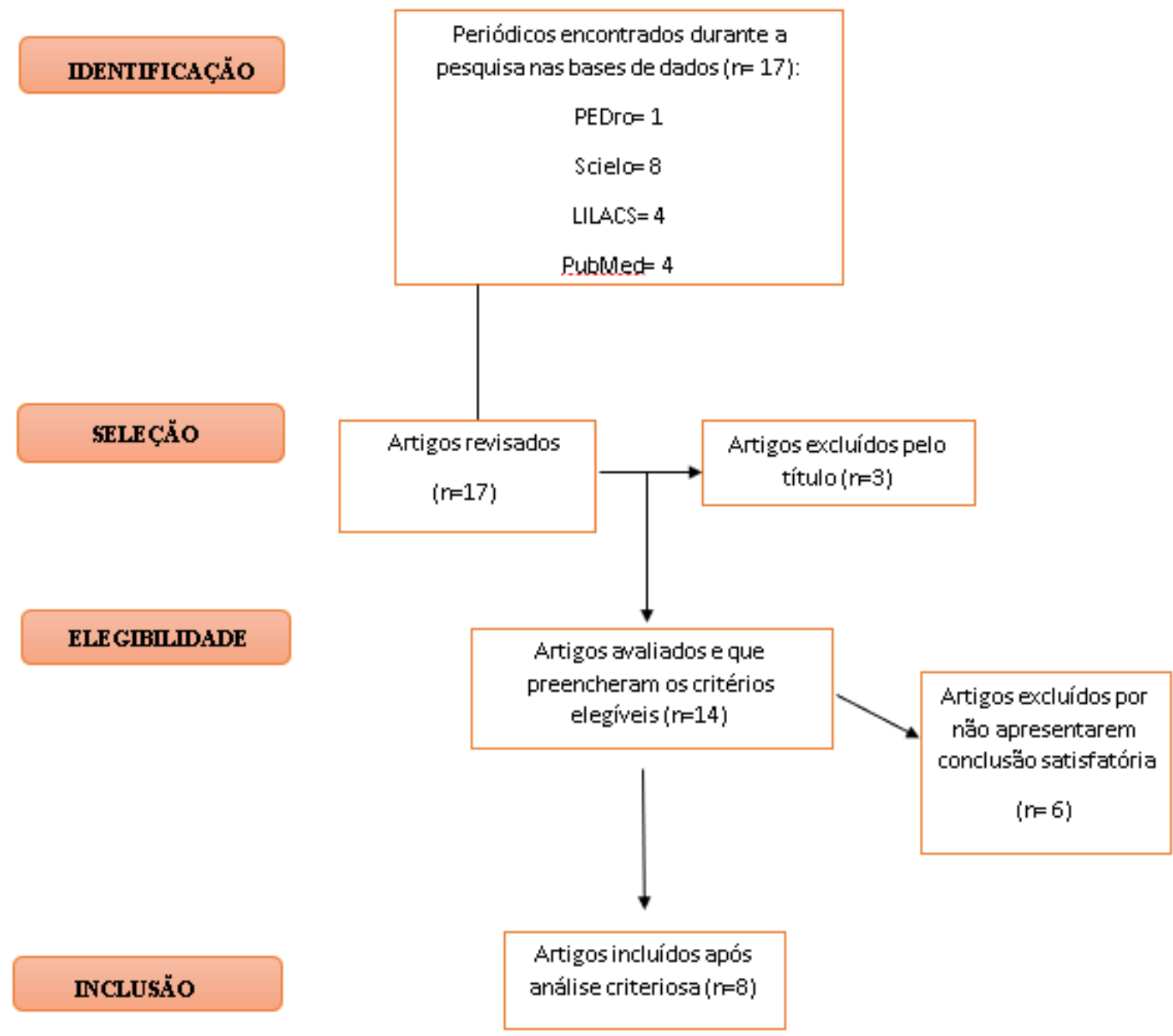

\section{CONCLUSÃO}

Diante disso, concluímos com essa revisão integrativa que a fisioterapia é indicada para pacientes durante a oxigenação por membrana extracorpórea, quando bem empregada e realizada por profissionais capacitados e equipe multidisciplinar alinhada. A fisioterapia motora e respiratória deve ser realizada o quanto antes para obtermos resultados satisfatórios e diminuir os efeitos adversos gerados pela internação prolongada. Evidenciamos que a mobilização precoce pode minimizar o delirium, diminuir o tempo de VMI e os efeitos deletérios da imobilização, melhorando a funcionalidade e capacidade do paciente de retornar para as atividades de vida diária (AVDs) o quanto antes. Entretanto, mais estudos devem ser realizados para confirmar os efeitos benéficos da fisioterapia durante a ECMO, e quais cuidados devem ser tomados para evitar colocar o paciente em risco durante a terapia. 


\section{V.10 N.1 (2022) ISSN: 2317-434X}

\section{REFERÊNCIAS}

BROMAN, Lars Mikael et al. Extracorporeal membrane oxygenation for COVID-19 during first and second waves. The Lancet Respiratory Medicine, 2021. DOI: 10.1016/S2213-2600(21)00262-9.

CARSWELL A, Roberts A, Rosenberg A, Zych B, Garcia $\mathrm{D}$, Simon A, et al. Mobilisation of patients with venovenous extracorporeal membrane oxygenation (VV ECMO): A case series. Eur J Heart Fail. 2017;19(Suppl2):26-7.

DE LIMA LAGES, Natália Coronel; TIMENETSKY, Karina Tavares. Recomendações para a atuação dos fisioterapeutas nos casos de oxigenação por membrana extracorpórea (ECMO). ASSOBRAFIR Ciência, v. 11, n. Supl1, p. 161-173, 2020.

FERREIRA, Daniele da Cunha et al. Segurança e potenciais benefícios da fisioterapia em adultos submetidos ao suporte de vida com oxigenação por membrana extracorpórea: uma revisão sistemática. Revista Brasileira de Terapia Intensiva, v. 31, p. 227 239, 2019. DOI: 10.5935/0103-507X.2019001
NAKASATO, Gislaine Rodrigues; LOPES, Juliana de Lima; LOPES, Camila Takao. Preditores de complicações da oxigenação por membrana extracorpórea. Revista Brasileira de Enfermagem, v. 73, 2020. DOI: 10.1590/0034-7167-2018-0666.

REHDER KJ, Turner DA, Hartwig MG, Williford WL, Bonadonna D, Walczak RJ Jr, et al. Active rehabilitation during extracorporeal membrane oxygenation, 2019. DOI:10.5935/0103-507X.20190017

SCHMIDT, Matthieu et al. Evolving outcomes of extracorporeal membrane oxygenation support for severe COVID-19 ARDS in Sorbonne hospitals, Paris. Critical Care, v. 25, n. 1, p. 1-11, 2021. DOI: 10.1186/s13054021-03780-6

WANG, Jing et al. Extracorporeal Membrane Oxygenation-Related Nosocomial Infection after Cardiac Surgery in Adult Patients. Brazilian Journal of Cardiovascular Surgery, 2021. DOI: $10.21470 / 1678-$ 9741-2020-0068. 